\title{
Blood pressure and body mass index: a comparison of the associations in the Caucasian and Asian populations
}

\begin{abstract}
Huynh L Quan ${ }^{1}$, Christopher L Blizzard ${ }^{1}$, Alison J Venn ${ }^{1}$, Au B Thuy ${ }^{1}$, Pham H Luc ${ }^{2}$ and James E Sharman ${ }^{1}$
A strong association between blood pressure (BP) and body mass index (BMI) has been observed in developed and developing countries. Whether there are differences in these associations between Caucasians and Asians remains unknown. Our objective was to compare the associations of BP with fatness measures in the Caucasian and Asian samples. The study used data from two population-based cross-sectional studies conducted using similar methodology: a survey in Australia in $1998-1999$ ( $n=832$ adults aged 25-64 years; 47\% male) and a survey in Vietnam in 2005 ( $n=1978$ adults aged 25-64 years; 46\% male). Participants completed questionnaires and attended clinics for physical measurements including BP and anthropometry. Linear regression was used for analysis. Independent of age, there were strong associations between BP indices and BMI in each sample, but the patterns of associations were different. Among Caucasians, pulse pressure (PP) increased with increasing BMI because the slope of systolic pressure with BMI exceeded the slope of diastolic pressure with BMI ( $P<0.001$ for both sexes). In contrast, among Asians, PP decreased with increasing BMI. Associations between BMI and BP are different between Caucasian and Asian populations. Among Asians, the stronger association of increasing BMI and diastolic BP, but not PP, suggests a different pathophysiology related to hypertension.

Hypertension Research (2012) 35, 523-530; doi:10.1038/hr.2011.219; published online 12 January 2012
\end{abstract}

Keywords: blood pressure; body fat; body mass index; pulse pressure; racial

\section{INTRODUCTION}

High blood pressure (BP) is strongly associated with an increased risk of cardiovascular disease, including heart disease and stroke, and is a major contributor to the total burden of death and disease worldwide. ${ }^{1} \mathrm{BP}$ is affected by genetic and lifestyle factors and is increased in those who are physically inactive, smokers, overweight or obese, have high dietary salt intakes or high alcohol consumption. ${ }^{2}$ Although much is known about the epidemiology of high BP in adults in developed countries, less is known about the prevalence and predictors of high BP in developing countries. In Vietnam, these issues have been investigated in a few published studies. ${ }^{3-6}$ Mirroring reasonably well the results of studies in Western countries, predictors of elevated BP identified in these studies include increasing age, male sex, tobacco smoking and alcohol consumption, indicators of overweight or body fatness, markers of low socioeconomic status including education and occupation, and marital status.

Obesity has been a serious public health problem in the Caucasian populations for years, and is now an emerging problem in the Asian populations ${ }^{7}$ including the Vietnamese. ${ }^{8}$ Positive associations between obesity and BP have been well established in both developed and developing countries. ${ }^{9}$ Several studies have compared the relationship of body composition and BP in black and white racial groups ${ }^{9}$ or between African and Asian populations. ${ }^{10}$ However, to our knowledge, no study has reported such a relationship in population-based samples of the Caucasian and Asian populations, which was the principal aim of this study. This is an important step to better understand the potential cardiovascular consequences of increasing numbers of overweight and obese individuals in the Asian populations.

\section{METHODS}

Study population

The study used data from two population-based cross-sectional studies conducted using similar methodology: a survey by the Burnie Take Heart (BTH) project of residents of Burnie (Australia) in 1998-1999 ( $n=832$ adults aged 25-64 years; $47 \%$ male; $69 \%$ response), and a survey of residents of Cantho (CT) (Vietnam), conducted in 2005 ( $n=1978$ adults aged 25-64 years; 46\% male; $74 \%$ response). The population of Burnie is predominantly Australianborn, Caucasian and English speaking. In 1996, when the BTH project commenced, only $0.03 \%$ of the population of Burnie was born overseas in a non-English speaking country. ${ }^{11}$ Cantho is the biggest city in the Mekong Delta, which is the far southern region of Vietnam and contains $20 \%$ of the country's population. ${ }^{12}$ The study participants of this survey comprised $92 \%$ Vietnamese and $8 \%$ were of Chinese, Khmer or another ethnicity.

Sampling procedures and methods of data collection for the BTH and CT surveys have been presented elsewhere. ${ }^{6,13}$ Both surveys had approximately 
equal numbers of participants within four age categories (25-34, 35-44, 45-54 and 55-64 years). In BTH, participants were selected by age- and sex-stratified random sampling from among those on the Electoral Roll of registered voters in Tasmania who had an address within the Burnie municipalities. In CT, participants were randomly selected from permanent residents in Cantho, by multistage sampling with age, sex and urban/rural stratification. Health volunteers, who are responsible for providing basic health services for an assigned local area, maintain and update the lists of the residents of the area regularly.

All participants provided written informed consent. In CT, those who could not sign provided verbal consent. The studies had approval from the Ethics Committees of the University of Tasmania (BTH) and the Can Tho University of Medicine and Pharmacy (CT).

\section{Measurements}

Trained staff obtained physical measurements of participants, including BP and anthropometry. In both surveys, BP was measured at the mid-point of the right upper arm, in an upright sitting position, with feet flat on the ground, back supported and no talking, after participants had rested for at least $5 \mathrm{~min}$. Correct cuff sizes were determined before measurement based on right upper arm circumference. Three readings, at least $30 \mathrm{~s}$ apart (after the cuff was completely deflated), were taken in $\mathrm{BTH}$, whereas a third reading was taken in CT only if there was a difference of $>25 \mathrm{~mm} \mathrm{Hg}$ for systolic BP (SBP) or $15 \mathrm{~mm} \mathrm{Hg}$ for diastolic BP (DBP) between the first two readings (in accordance with the STEPSwise approach to surveillance of non-communicable diseases (STEPS) manual ${ }^{14}$ in 2005). A mean of all measurements was used for analysis in both surveys. A Dinamap Vital Signs Monitor 1846SX (Critikon Company, Tampa, FL, USA) was used in BTH. This device was calibrated against a mercury sphygmomanometer before each week of clinic use. An Omron T9P digital automatic BP monitor (Omron Healthcare, Kyoto, Japan) was used in CT. This device was calibrated against a mercury sphygmomanometer before each day of clinic use.

Anthropometric measurements included weight, height, waist circumference and hip circumference. Each participant's weight was measured to the nearest $0.1 \mathrm{~kg}$, in bare feet without heavy clothing, using digital scales. Height was measured to the nearest $0.1 \mathrm{~cm}$, in bare feet without headwear, using a stadiometer. Waist circumference at the narrowest point between the lower costal border and the iliac crest, and hip circumference at the greatest posterior protuberance of the buttocks, were measured to the nearest $0.1 \mathrm{~cm}$ using a nonstretching constant tension tape.

Total cholesterol and plasma glucose were measured via standard laboratory procedure in venous blood in participants who had fasted for at least $9 \mathrm{~h}$ (BTH) and in capillary blood in participants who had fasted for at least $12 \mathrm{~h}$ using a Roche Diagnostics Accutrend Glucometer (Roche Group, Basel, Switzerland) (CT).

Participants in both surveys completed questionnaires on demographic characteristics, socio-economic factors and cardiovascular behavioral risk factors (smoking, alcohol consumption, diet and physical activity). In BTH, the questionnaire was based with minor modification on the questionnaire developed by the National Heart Foundation of Australia and Australian Institute of Health to determine the prevalence of cardiovascular risk factors in Australian adults. ${ }^{15}$ The standardised STEPS survey methodology developed by the World Health Organization ${ }^{14}$ was used in CT. Some locally relevant questions and response options were added to the STEPS questionnaire, in accordance with the STEPS protocols. ${ }^{14}$ Although similar in most other respects, the questionnaires differed in the items used to assess physical activity. The BTH questions addressed time spent on vigorous and less vigorous exercise, and on vigorous tasks at work and around the house, during the past 2 weeks. The STEPS questionnaire addressed time on moderate and vigorous activities in three domains (work, transport and leisure) during a typical week.

\section{Statistical analyses}

Linear regression models including covariates for age and (where necessary) the square of age were used to produce the trend lines. The differences by sex in mean levels of BP reported in Table 2 were assessed from a regression model that included a single covariate for sex, and the differences by sex in the association with age in Figures 1 and 2 were assessed from a regression model that also included product terms formed from the covariates for sex and age. The mean values of SBP, DBP, pulse pressure ( $\mathrm{PP}=\mathrm{SBPDBP}$ ) and the mean arterial pressure $(\mathrm{MAP}=1 / 3 \mathrm{SBP}+2 / 3 \mathrm{DBP})$ were calculated and reported in Table 2. For these analyses, as in the regression analyses, the BP measurements were transformed before analyses to remove skewness but all results are presented in the original units. Rank correlations of BP with indices of overweight and fatness presented in Supplementary Table 1 were calculated

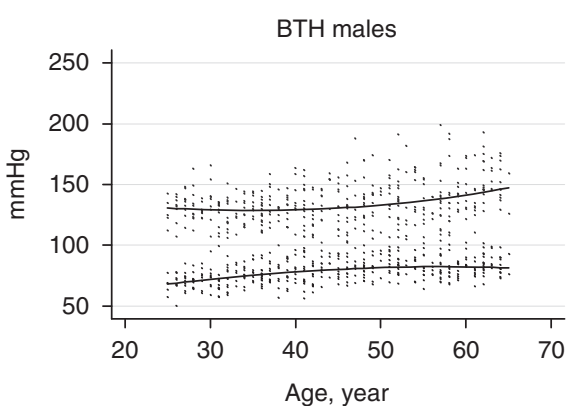

CT males

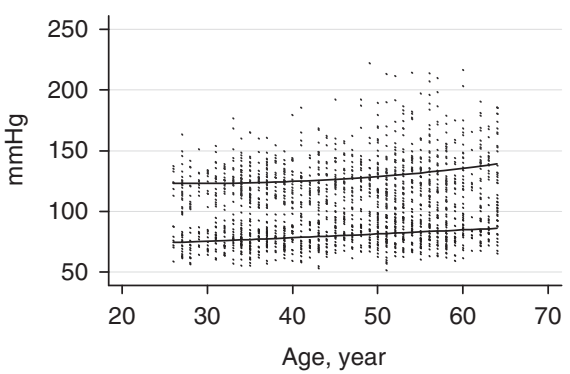

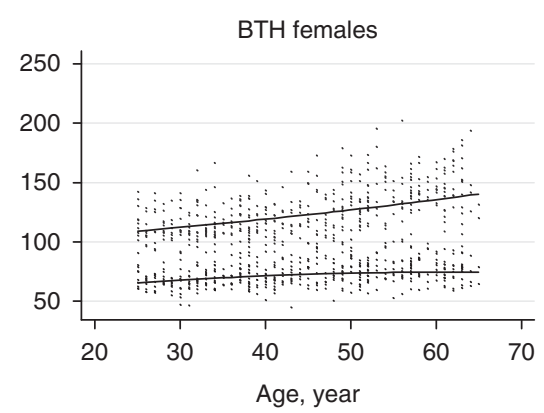

CT females

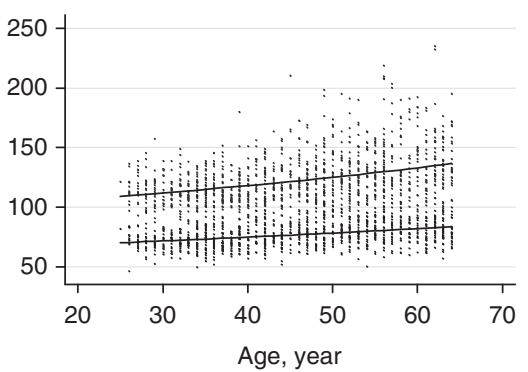

Figure 1 Associations of blood pressure and age, by population and sex. In each figure, the higher set of points and trend lines represent systolic blood pressure and the lower set represent diastolic blood pressure. BTH, Burnie Take Heart; CT, Cantho. A full color version of this figure is available at the Hypertension Research journal online. 

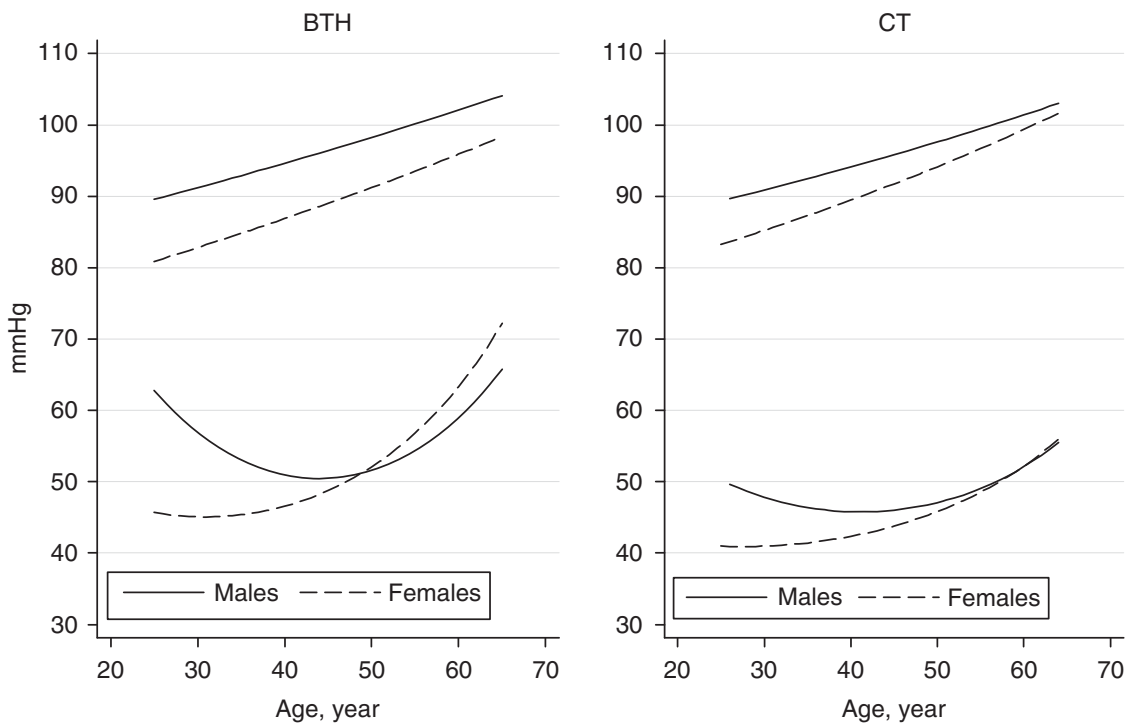

Figure 2 Trend lines depicting the association of mean arterial pressure (at top) and pulse pressure (at bottom) with age for men and women in the Burnie Take Heart (BTH) study and Cantho (CT) survey. A full color version of this figure is available at the Hypertension Research journal online.
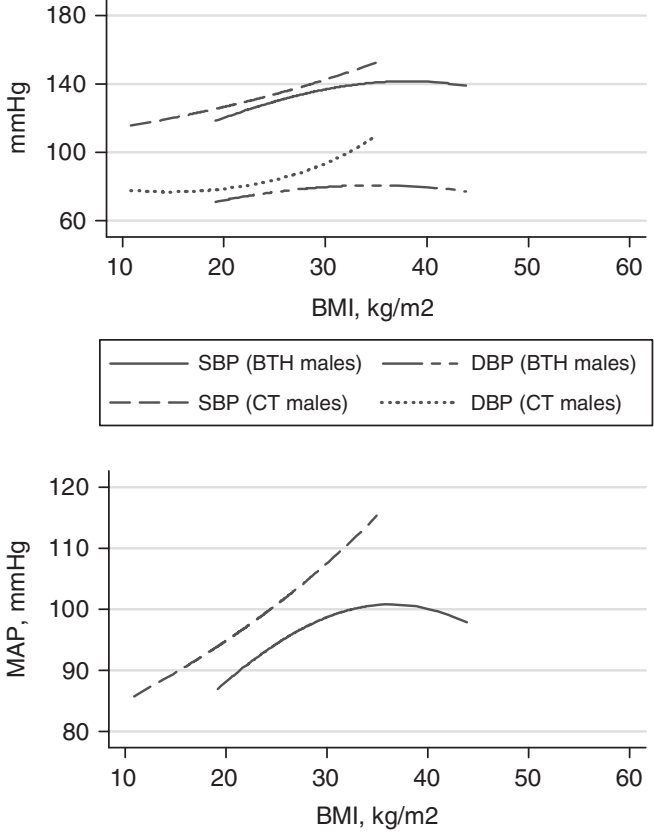

MAP (BTH males) - - MAP (CT males)
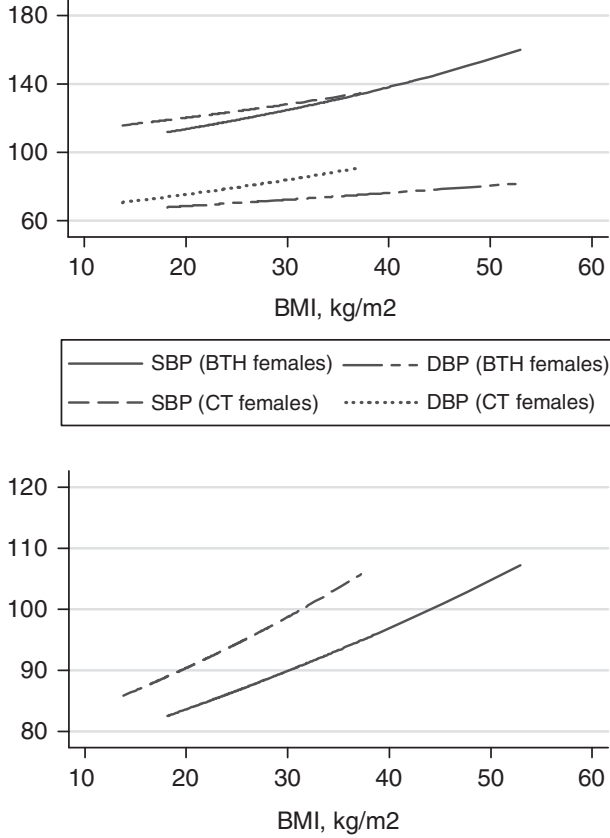

MAP (BTH females) - - - MAP (CT females)

Figure 3 Age-adjusted associations of three indices of blood pressure with body mass index, by population and sex. BMI, body mass index; BTH, Burnie Take Heart); CT, Cantho); DBP, diastolic blood pressure; MAP, mean arterial pressure; SBP, systolic blood pressure. A full color version of this figure is available at the Hypertension Research journal online.

by applying Pearson's correlation coefficient to the ranks of the variables with age as a covariate. The trend lines presented in Figures 3 and 4 were produced from linear regression models including as covariates linear predictors for age, body mass index (BMI) and the square of BMI (if required). The adjusted estimates reported in Supplementary Table 2 were produced by adding as covariates linear predictors for total years of smoking, weekly frequency of alcohol consumption, total metabolic equivalent-minutes) of physical activity and the binary indicator of fruit and vegetable consumption (two or more servings of fruit and five or more servings of vegetable per day), and calculating the estimated effect of an additional one unit $\left(1 \mathrm{~kg} \mathrm{~m}^{-2}\right)$ of BMI in the adjusted model. Further analyses of cholesterol were undertaken by including as covariates linear predictors for cholesterol and the product of cholesterol and age.

The analyses reported in Table 1, Figures 1 and 2 were unweighted. All other analyses were weighted to the World Standard Population ${ }^{16}$ to enable comparisons free of discrepancies due to the slight differences in the age distributions of the two samples. Specifically, this required us to attach a weight to each observation such that the age distribution of 25-34, 35-44, 45-54 and 55-64-year olds in each sample matched that of the World Standard Population.

Hypertension was defined as an average BP $\geqslant 140 / 90 \mathrm{~mm} \mathrm{Hg}$, or use of antihypertensive medication. BMI was calculated as weight $(\mathrm{kg})$ divided by 

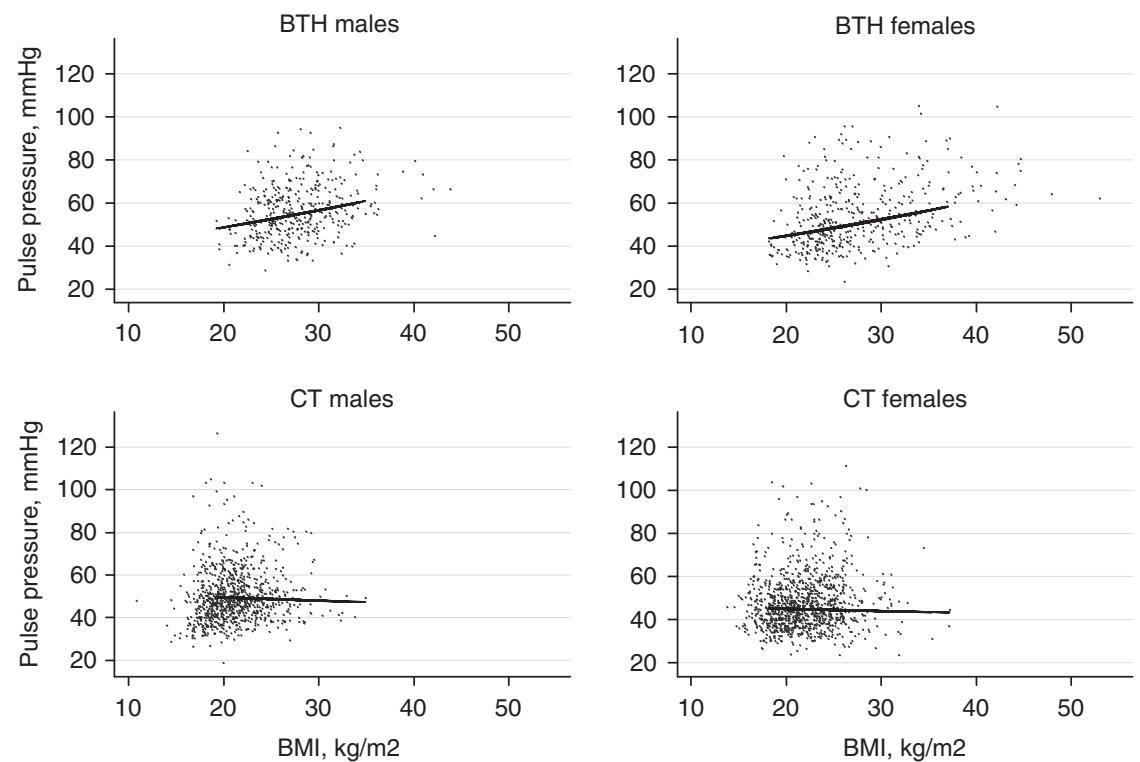

Figure 4 Age-adjusted associations of pulse pressure with body mass index, by population and sex. The trend lines are plotted over the range of overlapping BMI in the two populations: $19.2-34.9 \mathrm{~kg} \mathrm{~m}^{-2}$ for men, and $18.2-37.2 \mathrm{~kg} \mathrm{~m}^{-2}$ for women. BMI, body mass index; BTH, Burnie Take Heart); CT, Cantho. A full color version of this figure is available at the Hypertension Research journal online.

height squared $\left(\mathrm{m}^{2}\right)$. All participants who were taking antihypertensive medication at the time of study clinics were excluded from the analyses.

\section{RESULTS}

Characteristics of study participants are shown in Table 1. Participants were approximately equally distributed across age categories as a result of the sampling design. Mean ages were 44.6 (s.d.=11.6) and 45.8 $(\mathrm{s.d} .=10.5)$ years in BTH and CT, respectively. Most subjects in BTH had completed secondary school level education, but the majority in CT either had primary school as the highest level of education or had no formal education. A greater proportion of participants in BTH than in CT had values of BMI in the overweight or obese categories. Greater proportions of men than women were in these categories in BTH while the reverse was true in CT. The mean values of BMI, for men and women, respectively, were 27.6 (s.d.=3.9) and 27.4 (s.d.=5.8) in BTH, and 21.0 (s.d.=3.0) and $21.8\left(\right.$ s.d.=3.3) $\mathrm{kg} \mathrm{m}^{-2}$ in CT. The mean values of weight, waist circumference and waist-hip ratio mirrored these differences. Cigarette smoking was very common among men in CT, but rare among women there. Relatively more male than female participants consumed alcohol regularly, with consumption highest among BTH participants.

Figure 1 depicts associations of SBP and DBP with age, by sex and population. Younger women in these samples of 25-64 year olds had lower mean levels of both SBP and DBP than their male counterparts (as shown in Table 2), but this difference was diminished among older participants. Figure 2 displays trend lines depicting the association of MAP and PP with age. In both samples, younger men had higher mean values of MAP and PP than their female counterparts, but this difference was progressively diminished among older participants (BTH $P=0.114$ (MAP), $P<0.0001$ (PP); CT $P=0.005$ (MAP), $P<0.0001(\mathrm{PP}))$.

The mean values of four measures of BP are shown in Table 2, stratified by sex, age group and population. These sex and age groups were used in the stratified sampling design in each location. In each age group, mean SBP was higher among men in BTH than in CT, whereas mean DBP was generally higher among men in CT than in
BTH. This led to a higher mean level of PP among men in BTH than in CT. In general, men in BTH had marginally higher MAP than men in CT. Among women, a higher mean level of PP in BTH than in CT was also observed. Although there were approximately equal mean levels of SBP among women in the two surveys, higher mean levels of DBP were observed in CT. Among women, mean levels of MAP were always higher for CT than for BTH participants in each of the four age groups.

Age-adjusted rank correlations of BP with various indices of overweight and fatness, stratified by sex and population, are shown in Supplementary Table 1. There were moderate associations between body fatness and BP measures among men and women with the exception of PP among CT women, for which the associations were weak. The strength of correlations with BP was similar across body fatness measures and, in subsequent analyses we present data for BMI only, given that this is a commonly used index in research and clinical practice.

Figure 3 illustrates the estimated relationships of SBP, DBP and MAP with BMI, by sex and population, after adjusting for age. In each population, the trends of the relationships were similar for men and women. MAP increased with BMI among BTH women, but declined at high and increasing levels of BMI among BTH men. For the CT sample, MAP increased with BMI because both SBP and DBP increased with BMI.

The values of PP plotted against BMI in Figure 4 showed that PP increased with BMI among the BTH samples, but not among the CT samples. These differing results for PP were not due to different distributions of BMI in the two populations. Restricting the analyses to the $95.9 \%$ of BTH men and $71.3 \%$ of CT men whose BMI fell in the 19.2 (BTH minimum) to 34.9 (CT maximum) $\mathrm{kg} \mathrm{m}^{-2}$ range, and to the $92.7 \%$ of $\mathrm{BTH}$ women and $87.5 \%$ of CT women whose BMI fell in the 18.2 (BTH minimum) to 37.2 (CT maximum) $\mathrm{kg} \mathrm{m}^{-2}$ range, all relationships between $\mathrm{PP}$ and BMI were linear and positive for BTH participants (men $P<0.001$, women $P<0.001$ ) but negative for CT participants (men $P=0.324$, women $P=0.358)$. 
Table 1 Characteristics of participants in Burnie Take Heart and Cantho surveys

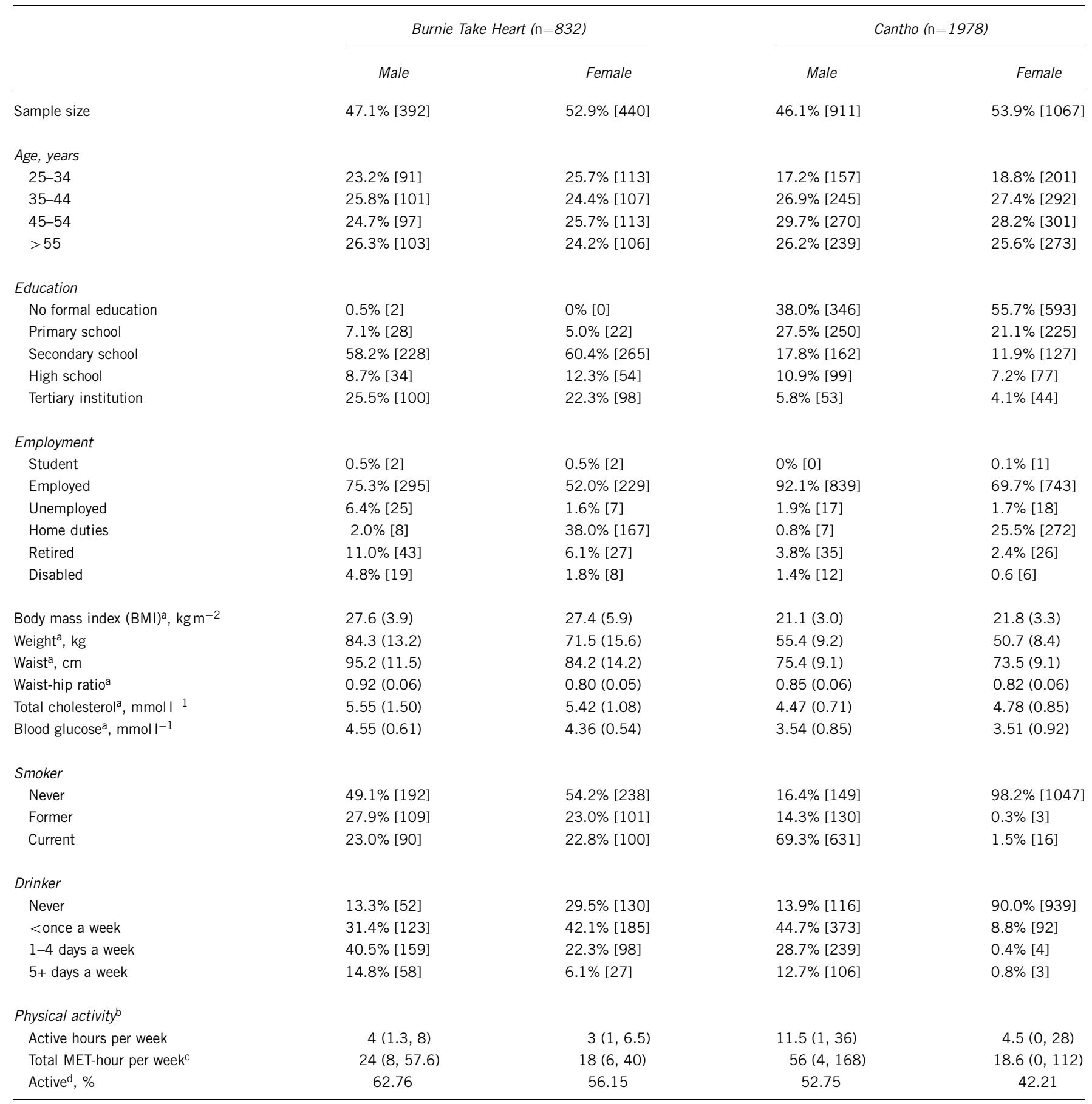

The data displayed are in percentages and numbers, unless otherwise stated.

aData shown are mean (s.d.).

bData shown are median (interquartile range).

cTotal metabolic equivalent task unit (MET-hour) per week

${ }^{\mathrm{d} A c t i v e}$ is defined as having at least equivalent $150 \mathrm{~min}$ of moderate activities per week.

We investigated whether these relationships were independent of other factors that may be associated with BP, such as years of smoking, weekly frequency of alcohol intake, dietary consumption of fruit and vegetables, and physical activity. However, adjusting for these factors had little impact (as shown in Supplementary Table 2).

To determine whether differences in metabolic parameters could account for the different patterns of association between PP and BMI in the two populations, we adjusted the associations for fasting blood total cholesterol and glucose concentrations. Cholesterol was positively associated with PP among BTH women $(P=0.018)$ and negatively associated with PP among CT men $(P=0.04)$, but adjusting for it had only a marginal effect on the estimated association of PP with BMI in each population (data not shown). Glucose was not a significant predictor of BP among BTH partici- 
Table 2 Mean values of blood pressure for subjects classified by sex and age group, by population

\begin{tabular}{|c|c|c|c|c|}
\hline & \multicolumn{4}{|c|}{ Age group, year } \\
\hline & $25-34$ & $35-44$ & $45-54$ & $55-65$ \\
\hline & Mean (s.d.) & Mean (s.d.) & Mean (s.d.) & Mean (s.d.) \\
\hline \multicolumn{5}{|l|}{$B T H$} \\
\hline \multicolumn{5}{|l|}{ Male } \\
\hline SBP & $129.2(12.6)$ & $130.9(14.5)$ & $136.3(18.7)$ & 142.1 (19.9) \\
\hline DBP & $71.7(8.2)$ & $77.5(9.4)$ & $83.3(10.0)$ & $82.3(8.9)$ \\
\hline PP & $56.8(10.4)$ & 52.5 (9.9) & $52.1(11.4)$ & $59.7(14.7)$ \\
\hline MAP & $91.0(8.6)$ & $95.5(10.3)$ & $101.1(12.4)$ & $102.2(11.7)$ \\
\hline \multicolumn{5}{|l|}{ Female } \\
\hline SBP & $112.8(12.8)$ & $116.8(13.5)$ & $130.6(20.1)$ & $143.0(19.4)$ \\
\hline DBP & $67.5(8.6)$ & $70.9(8.8)$ & $75.8(11.1)$ & 76.5 (9.8) \\
\hline PP & $45.4(8.8)$ & $45.6(8.4)$ & $54.3(14.2)$ & 66.5 (16.0) \\
\hline MAP & $82.6(9.4)$ & 86.4 (9.9) & $94.1(13.1)$ & $98.6(11.5)$ \\
\hline \multicolumn{5}{|l|}{$C T$} \\
\hline \multicolumn{5}{|l|}{ Male } \\
\hline SBP & $124.3(12.9)$ & $124.0(15.2)$ & $130.4(19.4)$ & $135.6(22.4)$ \\
\hline DBP & $75.5(9.8)$ & $77.4(11.5)$ & $83.9(13.1)$ & $83.2(12.4)$ \\
\hline PP & $48.1(8.9)$ & $46.0(8.8)$ & $47.4(11.4)$ & $51.7(14.0)$ \\
\hline MAP & $91.9(10.1)$ & $92.9(12.0)$ & $99.4(14.7)$ & $100.8(15.0)$ \\
\hline \multicolumn{5}{|l|}{ Female } \\
\hline SBP & $113.9(12.0)$ & $115.9(13.8)$ & $127.1(19.0)$ & $135.5(23.0)$ \\
\hline DBP & $72.4(8.3)$ & 73.7 (9.9) & 80.3 (11.9) & 81.9 (12.5) \\
\hline PP & $41.2(7.3)$ & $42.0(7.6)$ & $46.8(10.9)$ & $53.5(15.3)$ \\
\hline MAP & $86.4(9.1)$ & $87.9(10.8)$ & $95.9(13.7)$ & $99.9(15.2)$ \\
\hline
\end{tabular}

Abbreviations: BTH, Burnie Take Heart; CT, Cantho; DBP, diastolic blood pressure; MAP, mean arterial pressure; $\mathrm{PP}$, pulse pressure; $\mathrm{SBP}$, systolic blood pressure.

pants, but was a significant positive predictor of MAP among CT participants (men $P<0.001$, women $P=0.072$ ). However, adjusting for glucose only marginally reduced the positive linear slope between MAP and BMI among CT participants (data not shown).

Finally, although the proportion with hypertension among BTH men $(37.1 \%$, 95\% confidence interval (CI): $32.7 \%, 42.0 \%)$ and BTH women $(28.2 \%, 95 \%$ CI: $24.2 \%, 32.9 \%)$ exceeded the respective proportion among CT men (30.8\%, 95\% CI: 28.0, 33.8\%) and CT women $(21.3 \%$, 95\% CI: $18.9,24.0 \%)$, only $13.8 \%$ of the hypertensives in CT reported taking antihypertensive medication compared to $33.1 \%$ in BTH. These subjects were excluded from analyses but, in the remaining sample, the proportion with non-medicated SBP $>180 \mathrm{~mm} \mathrm{Hg}$ and/or non-medicated DBP $>110 \mathrm{~mm} \mathrm{Hg}$ among CT subjects $(3.7 \%)$ was more than twice that among BTH subjects (1.8\%). Particularly for SBP, this can be observed in Figure 1. To investigate whether this greater proportion of high values among the CT sample had influenced our results, we repeated the analyses further excluding non-medicated subjects with SBP $>180 \mathrm{~mm} \mathrm{Hg}$ and/or DBP $>110 \mathrm{~mm} \mathrm{Hg}$. No marked differences were found (data not shown).

\section{DISCUSSION}

This study examined the associations of BP with age and body composition in population-based samples of the Caucasians and
Asians. The main findings of the study were significant differences in the relationships between PP and body composition for Caucasians compared with Asians. The relationships between BP and age, and between other indices of BP and body composition, were generally similar between populations. Although further work is required, these observations provide new information regarding race, body composition and BP that may help inform public health planning in the future.

Although there were major discrepancies in the associations between PP and BMI, it was interesting to find similarities in the two populations for the age-BP relationships. The fall (or leveling off) of DBP together with the rise of SBP and PP with increasing age (particularly after the 5th decade) among both BTH and CT participants in these cross-sectional associations is consistent with agerelated abnormalities of the arterial wall resulting in large artery stiffness. ${ }^{17,18}$ This effect has been demonstrated to increase cardiovascular risk, ${ }^{19}$ probably owing to concomitant raised central BP. We also observed a lower mean PP in young women than young men, and this sex difference gradually narrowed and reversed at the fifth and sixth decade in BTH and CT, respectively. These findings are consistent with results from other studies ${ }^{18,20}$ and suggest that-regardless of differences in race, culture and demography-Caucasian and Asian people probably have similar physiology in relation to vascular aging.

Increasing BMI was associated with significant increases in PP among participants in BTH, but not among participants in CT. The difference was not due to different distributions of BMI in the two populations, because around $80 \%$ of the men and $90 \%$ of the women in the two populations had BMI in the same range, and we estimated the relationships among participants in the same range of BMI. The difference in PP between the two populations in response to increasing body composition suggests the possibility of different pathophysiology related to hypertension in Caucasians and Asians. In BTH, higher PP with increasing BMI may be indicative of increased large artery stiffness, similar to the association between higher PP and increasing age after about 50 years. Conversely, in CT, we observed increases of both SBP and DBP with associated increase in MAP but not in PP. This type of hypertension in CT is more typical of essential hypertension, in which both SBP and DBP are raised. Although speculative, this may be a result of increased circulatory volume or peripheral vascular resistance, possibly due to high-sodium intake, ${ }^{21}$ rather than being due to increased large artery stiffness per se. This conclusion is not necessarily consistent with some studies that have reported increased arterial stiffness in the Asians compared with Caucasians. ${ }^{22-25}$ These studies included only participants of South Asian origin, who are known to have higher rates of diabetes and premature coronary artery disease ${ }^{26,27}$ than the South-East Asian participants in this study. Other studies are required to elucidate causes of these racial differences in BP.

Some studies ${ }^{28,29}$ have also shown that at any value of SBP, cardiovascular risk is higher with lower DBP. Whether there is any difference in overall cardiovascular risk associated with increased BMI and the consequent BP differences between populations is unknown. Importantly, adjustment for other potential confounders such as tobacco smoking, alcohol consumption, fruit and vegetable consumption, and physical activity showed negligible effects on the relationship of BP and BMI. Whatever the reasons for the observed differences between these Caucasian and Asian populations, it is reasonable to conclude that these modifiable lifestyle behaviors did not have a major role. Of course the BMI-BP differences between Caucasians and Asians might be explained by genetic factors or other unmeasured lifestyle or environmental influences. 
A number of mechanisms by which obesity might contribute to vascular stiffness have been proposed. In obesity, macrophages accumulate in adipose tissue. ${ }^{30}$ This is believed to lead to the chronic state of low-grade inflammation (vascular and systemic) and endothelial dysfunction in obese people. ${ }^{31}$ Other artery-stiffening mechanisms of obesity may work through the functions of hormones such as insulin and leptin. An excess of circulatory insulin will promote sodium retention by increasing tubular reabsorption, ${ }^{32}$ stimulate sympathetic activity, ${ }^{33}$ proliferate vascular smooth muscle cells, ${ }^{34}$ promote endothelial dysfunction and impair vasodilator activity. ${ }^{35}$ Another possible mechanism of obesity-induced arterial stiffness is hyperleptinemia, which is also known to stimulate the sympathetic nervous system $^{36}$ and induce endothelial dysfunction. ${ }^{37}$

Hypercholesterolemia is also associated with increased large artery stiffness in Caucasians ${ }^{38}$ and this is known to affect peripheral BP. In this study, fasting serum total cholesterol was a significant positive predictor of PP in BTH women, though adjusting for total cholesterol had negligible effects on the relationship of PP and BMI. Similarly, fasting blood glucose was positively associated with MAP among CT participants, but had a negligible role in the relationship of MAP and BMI. Therefore, it seems reasonable to suggest that the difference in associations of BP and BMI between Caucasians and Asians observed in this study was not due to serum total cholesterol or glucose.

A limitation of this study was the cross-sectional design. Also there was a lack of information on sodium intake, which is known to be associated with hypertension and arterial stiffness. Although sodium consumption has been greatly reduced in western countries due to high awareness of cardiovascular risks, Asian countries generally have higher mean sodium intakes. ${ }^{21}$ This might help to explain the differences observed in this study between Caucasians and Asians. Using different BP devices in the two surveys would be a limitation if there were systematic errors between the two devices in measuring SBP and/or DBP, but only if these errors influenced the estimated relationships between PP and BMI. With the effect of minimizing this possibility, each BP device was calibrated against a standard mercury sphygmomanometer before each clinic examination for accuracy.

In conclusion, the principal finding in this study was that independent of conventional risk factors (for example, age, smoking, alcohol, diet and physical inactivity), BMI was positively associated with PP (and possibly large artery stiffness) among Caucasians, but not among Asians. This may be an important observation because increased $\mathrm{PP}$ is an independent predictor of myocardial infarction, congestive heart failure and cardiovascular death, even in hypertensive patients undergoing successful antihypertensive drug therapy. ${ }^{39}$ Our findings provide additional evidence for the importance of weight reduction to improve cardiovascular risk. The difference in associations of BMI and BP between Caucasians and Asians suggests a different pathophysiology related to hypertension and the possibility that different approaches to prevention and treatment of hypertension might be applied to the two populations. Future studies could perhaps focus on genetic and environmental factors to answer this important question.

\section{CONFLICT OF INTEREST}

The authors declare no conflict of interest.

\section{ACKNOWLEDGEMENTS}

The Burnie Take Heart project was funded by grants from the Commonwealth Department of Health and Aged Care, Australia. Data collection of the Cantho survey was funded by The Atlantic Philanthropies, Inc., USA.
1 Murray CJ, Lopez AD. Mortality by cause for eight regions of the world: Global Burden of Disease Study. Lancet 1997; 349: 1269-1276.

2 Kornitzer M, Dramaix M, De Backer G. Epidemiology of risk factors for hypertension: implications for prevention and therapy. Drugs 1999; 57: 695-712.

3 Van Minh H, Thanh LC, Thi BN, do Trinh T, Tho TD, Valensi P. Insulinaemia and slight overweight: the case of Vietnamese hypertensives. Int J Obes Relat Metab Disord 1997; 21: 897-902.

4 Minh HV, Byass P, Chuc NT, Wall S. Gender differences in prevalence and socioeconomic determinants of hypertension: findings from the WHO STEPS survey in a rural community of Vietnam. J Hum Hypertens 2006; 20: 109-115.

5 Hoang VM, Byass P, Dao LH, Nguyen TK, Wall S. Risk factors for chronic disease among rural Vietnamese adults and the association of these factors with sociodemographic variables: findings from the WHO STEPS survey in rural Vietnam, 2005. Prev Chronic Dis 2007; 4: A22.

6 Pham LH, Au TB, Blizzard L, Truong NB, Schmidt MD, Granger RH, Dwyer T. Prevalence of risk factors for non-communicable diseases in the Mekong Delta, Vietnam: results from a STEPS survey. BMC Public Health 2009; 9: 291.

7 Ramachandran A, Snehalatha C. Rising burden of obesity in Asia. J Obes 2010; 2010: pii 868573.

8 Tuan NT, Tuong PD, Popkin BM. Body mass index (BMI) dynamics in Vietnam. Eur J Clin Nutr 2008; 62: 78-86.

9 Doll S, Paccaud F, Bovet P, Burnier M, Wietlisbach V. Body mass index, abdominal adiposity and blood pressure: consistency of their association across developing and developed countries. Int J Obes Relat Metab Disord 2002; 26: 48-57.

10 Tesfaye F, Nawi NG, Van Minh H, Byass P, Berhane Y, Bonita R, Wall S. Association between body mass index and blood pressure across three populations in Africa and Asia. J Hum Hypertens 2007; 21: 28-37.

111996 Census of Population and Housing: Burnie and Devenport SuburbsAustralian Bureau of Statistics; 2001 (updated 3 July 2008; cited 7 March 2011); Available from: http://www.abs.gov.au/ausstats/abs@.nsf/ProductsbyReleaseDate/11DAAF72D3D02EB2CA25739F000E542F?OpenDocument.

12 General Statistics Office of Vietnam. Population and Population Density in 2009 by Province. General Statistics Office of Vietnam: Hanoi, (updated 2009; cited 5 January 2011); Available from: http://www.gso.gov.vn/default_en.aspx?tabid=467\&idmid= 3\&ItemID=988 2009.

13 Schmidt MD, Blizzard CL, Venn AJ, Cochrane JA, Dwyer T. Practical considerations when using pedometers to assess physical activity in population studies: lessons from the Burnie Take Heart Study. Res Q Exerc Sport 2007; 78: 162-170.

14 World Health Organization. The STEPS Manual. World Health Organization: Geneva, 2008 (updated 13 June 2008; cited 17 Dec 2010); Available from: http:// www.who.int/chp/steps/riskfactor/en/index.html.

15 National Heart Foundation of Australia and Australian Institute of Health. Risk Factor Prevalence Study: Survey no 3 1989. National Heart Foundation of Australia and Australian Institute of Health: Canberra, 1990.

16 Breslow NE, Day NE. Rates and Rate Standardization. In: Breslow NE, Day NE (eds). Statistical Methods in Cancer Research, Vol II, The Design and Analysis of Cohort Studies (IARC Scientific Publications No 82). International Agency for Research on Cancer: Lyon, 1987, 48-79.

17 Milch RA. Matrix properties of the aging arterial wall. Monogr Surg Sci 1965; 2: 261-342.

18 Franklin SS, Gustin IV W, Wong ND, Larson MG, Weber MA, Kannel WB, Levy D. Hemodynamic patterns of age-related changes in blood pressure. The Framingham Heart Study. Circulation 1997; 96: 308-315.

19 Vlachopoulos C, Aznaouridis K, O'Rourke MF, Safar ME, Baou K, Stefanadis C. Prediction of cardiovascular events and all-cause mortality with central haemodynamics: a systematic review and meta-analysis. Eur Heart J 2010; 31: 1865-1871

20 Skurnick JH, Aladjem M, Aviv A. Sex differences in pulse pressure trends with age are cross-cultural. Hypertension 2010; 55: 40-47.

21 Brown IJ, Tzoulaki I, Candeias V, Elliott P. Salt intakes around the world: implications for public health. Int J Epidemiol 2009; 38: 791-813.

22 Pinto ES, Mensah R, Meeran K, Cameron JD, Murugaesu N, Bulpitt CJ, Rajkumar C. Peripheral arterial compliance differs between races: comparison among Asian, Afro-Caribbeans, and white Caucasians with type 2 diabetes. Diabetes Care 2005; 28: 496.

23 Din JN, Ashman OA, Aftab SM, Jubb AW, Newby DE, Flapan AD. Increased arterial stiffness in healthy young South Asian men. J Hum Hypertens 2006; 20: 163-165.

24 Gunarathne A, Patel JV, Gammon B, Hughes EA, Lip GY. Impact of mean arterial blood pressure on higher arterial stiffness indices in South Asians compared to white Europeans. J Hypertens 2008; 26: 1420-1426.

25 Rezai MR, Wallace AM, Sattar N, Finn JD, Wu FC, Cruickshank JK. Ethnic differences in aortic pulse wave velocity occur in the descending aorta and may be related to vitamin D. Hypertension 2011; 58: 247-253.

26 Gupta M, Brister S. Is South Asian ethnicity an independent cardiovascular risk factor? Can J Cardiol 2006; 22: 193-197.

27 Enas EA, Mohan V, Deepa M, Farooq S, Pazhoor S, Chennikkara H. The metabolic syndrome and dyslipidemia among Asian Indians: a population with high rates of diabetes and premature coronary artery disease. J Cardiometab Syndr 2007; 2: 267-275.

28 Benetos A, Zureik M, Morcet J, Thomas F, Bean K, Safar M, Ducimetiere P, Guize L. A decrease in diastolic blood pressure combined with an increase in systolic blood 
pressure is associated with a higher cardiovascular mortality in men. J Am Coll Cardiol 2000; 35: 673-680.

29 Blacher J, Staessen JA, Girerd X, Gasowski J, Thijs L, Liu L, Wang JG, Fagard RH, Safar ME. Pulse pressure not mean pressure determines cardiovascular risk in older hypertensive patients. Arch Intern Med 2000; 160: 1085-1089.

30 Cancello R, Tordjman J, Poitou C, Guilhem G, Bouillot JL, Hugol D, Coussieu C, Basdevant A, Bar Hen A, Bedossa P, Guerre-Millo M, Clement K. Increased infiltration of macrophages in omental adipose tissue is associated with marked hepatic lesions in morbid human obesity. Diabetes 2006; 55: 1554-1561.

31 Yudkin JS, Stehouwer CD, Emeis JJ, Coppack SW. C-reactive protein in healthy subjects: associations with obesity, insulin resistance, and endothelial dysfunction: a potential role for cytokines originating from adipose tissue? Arterioscler Thromb Vasc Biol 1999; 19: 972-978.

32 Rocchini AP, Key J, Bondie D, Chico R, Moorehead C, Katch V, Martin M. The effect of weight loss on the sensitivity of blood pressure to sodium in obese adolescents. $N$ Engl J Med 1989; 321: 580-585.
33 Anderson EA, Hoffman RP, Balon TW, Sinkey CA, Mark AL. Hyperinsulinemia produces both sympathetic neural activation and vasodilation in normal humans. J Clin Invest 1991; 87: 2246-2252.

34 DeFronzo RA, Ferrannini E. Insulin resistance. A multifaceted syndrome responsible for NIDDM, obesity, hypertension, dyslipidemia, and atherosclerotic cardiovascular disease. Diabetes Care 1991; 14: 173-194.

35 Kim JA, Montagnani M, Koh KK, Quon MJ. Reciprocal relationships between insulin resistance and endothelial dysfunction: molecular and pathophysiological mechanisms. Circulation 2006; 113: 1888-1904.

36 Rahmouni K, Haynes WG, Mark AL. Cardiovascular and sympathetic effects of leptin. Curr Hypertens Rep 2002; 4: 119-125.

37 Korda M, Kubant R, Patton S, Malinski T. Leptin-induced endothelial dysfunction in obesity. Am J Physiol Heart Circ Physiol 2008; 295: H1514-H1521.

38 Wilkinson I, Cockcroft JR. Cholesterol, lipids and arterial stiffness. Adv Cardiol 2007; 44: 261-277.

39 Safar ME. Systolic blood pressure, pulse pressure and arterial stiffness as cardiovascular risk factors. Curr Opin Nephrol Hypertens 2001; 10: 257-261.

Supplementary Information accompanies the paper on Hypertension Research website (http://www.nature.com/hr) 